\title{
Endodontic Treatment of Permanent Mandibular First Molar with 4 Roots and 5 Canals-Clinical Case Reports
}

\section{Sravana Laxmi Penumaka}

Government Dental College and Hospital, Vijayawada, Andhra Pradesh, India

*Corresponding author: Sravana Laxmi Penumaka, Government Dental College and Hospital, Vijayawada, Andhra Pradesh, India, Tel: +91 9701930787; E-mail: sravanipenumaka7@gmail.com

Received date: December 19, 2017; Accepted date: January 08, 2018; Published date: January 15, 2018

Copyright: (c) 2018 Penumaka SL. This is an open-access article distributed under the terms of the Creative Commons Attribution License, which permits unrestricted use, distribution, and reproduction in any medium, provided the original author and source are credited.

\begin{abstract}
Endodontic management of mandibular molars is a challenging task due to its varied morphology of roots and root canals. A mandibular permanent first molar with additional buccal root (Radix paramolaris) and additional distal root (Radix Entomolaris) is an example of its varied anatomy. A successful management of atypical root canal configurations is an important aspect in determining the success rate of endodontic therapy. The detail knowledge of the root morphology and canal anatomy allows the clinician for accurate location of the extra roots and canals and accordingly the refinement of the access cavity for the stress free entry of complex anatomy. Hence, for a successful endodontic therapy, clinician must be aware of the external and internal anatomic variations. The aim of these clinical case reports is to present and describe the unusual presence of two separate mesial roots, distal roots and 5 root canals in permanent mandibular first molar diagnosed during routine endodontic therapy.
\end{abstract}

Keywords Anatomical variations; Four rooted permanent mandibular first molar (Radix entoparamolaris); Mandibular first molar; Radix entomolaris; Radix paramolaris

\section{Introduction}

The prevention or ameliorating of an endodontic pathology relies on a thorough chemo mechanical cleaning of root canals prior to obturation to produce fluid-tight seal. But, the existence of extra roots and root canals if not recognized, it could prompt an endodontic failure [1].Therefore cognizance and assumption of the presence of uncommon roots and root canal morphology can contribute to the triumphant outcome of the root canal treatment.

The first permanent mandibular molar usually presents two separated roots: one mesial and the other distal [2]. It displays various anatomical variations [3]. A noteworthy variation of the two rooted morphology in the mandibular first molar is the presence of supernumerary root found distolingually, mentioned in the literature by Carabelli [4], as RADIX ENTOMOLARIS (RE) [5]. The supernumerary root present mesiobuccally is known as RADIX PARAMOLARIS. Carlsen and Alexandersen [6,7] described the diagnosis of external morphology of the additional buccal or lingual root.

\section{Case Reports}

\section{Case 1}

A 15 year old boy came to the Department of Conservative Dentistry \& Endodontics with the main complaint of pain in the right lower back region of mouth since five days. History revealed intermittent, throbbing pain localized to 46. Clinical examination revealed the presence of decayed right mandibular 1st molar 46. Tenderness is present on vertical percussion. The mobility of the tooth is within physiologic limits and the vitality tests revealed the tooth to be non-vital. The medical history of the patient was insignificant (Figure 1).

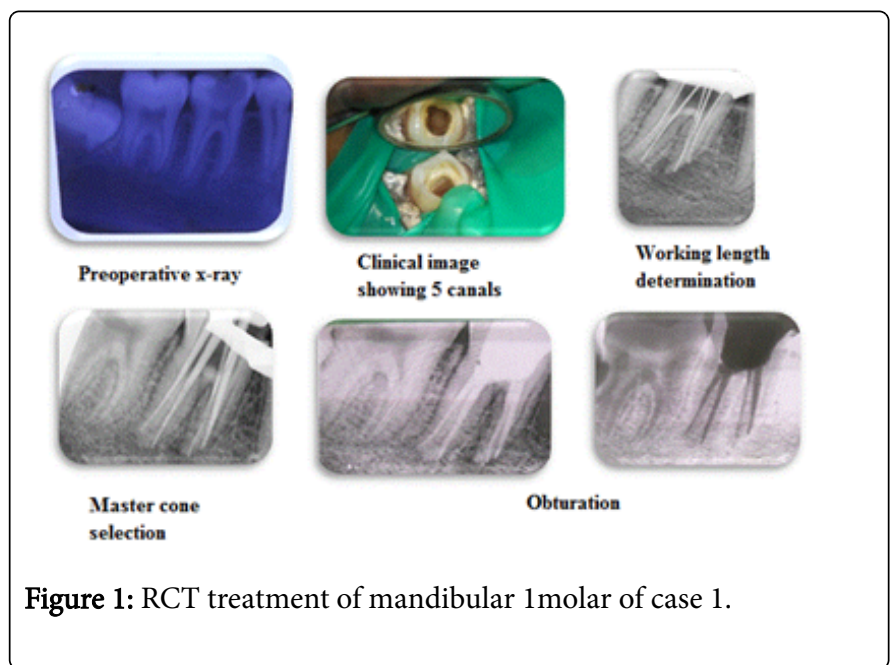

\section{Case 2}

A 15 year old girl reported to the Department of Conservative Dentistry \& Endodontics with the chief complaint of pain in the lower right back region of mouth since five days. Clinical examination revealed the presence of temporary restoration in relation to right mandibular 1st molar 46. Tenderness is present on vertical percussion. The mobility of the tooth is within physiologic limits. The medical history of the patient was noncontributory. After removing the temporary restoration mesiolingual perforation was detected and it was repaired with biodentine (Figure 2). 


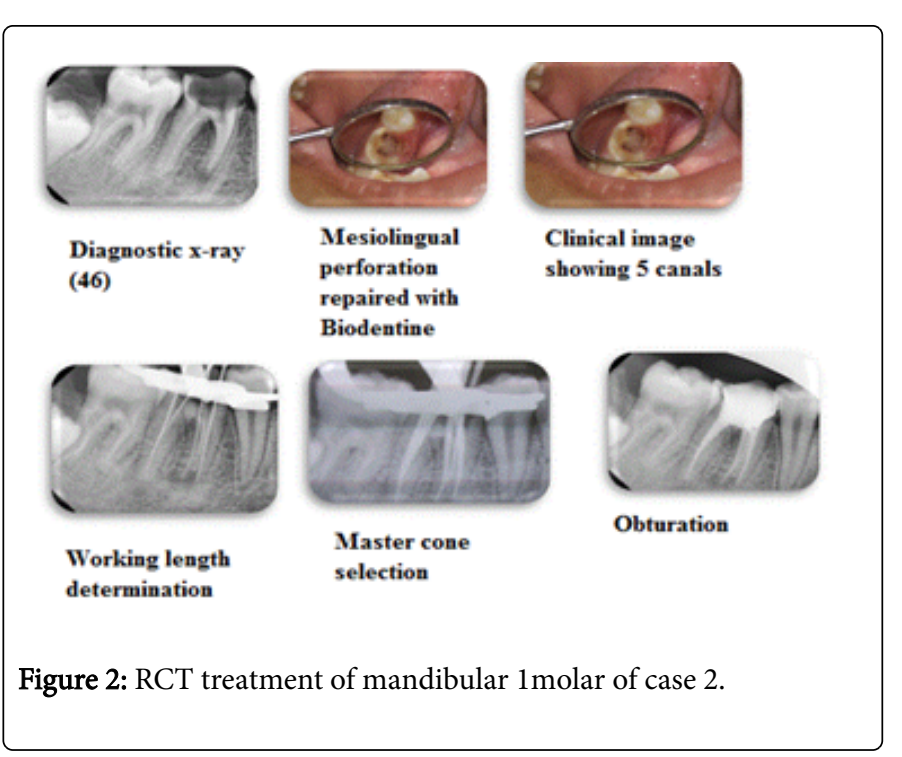

\section{Radiographic Examination}

A thorough radiographic assessment of the diagnostic radiographs of 2 cases demonstrates that an indistinct view or outline of both distal and mesial roots and root canals, indicates the existence of extra (hidden) roots. The second radiograph has been taken from a mesial and distal angle (20 degrees) to conventional angulation. These radiographs reveal the hidden roots of REM and RPM. Thickening of the pdl space, inter radicular radiolucency and periapical radiolucencies with irregular borders indicates that chronic periapical abscess in relation to mandibular first molar in 3 cases.

The conventional access cavity was modified from a triangular (rectangular) to a trapezoid form to improve the access to the additional canals. Careful investigation of the pulp chamber with a $\mathrm{K}$ file number 15 (Dentsply) was used to negotiate the canals. Root canal orifices were named according to the terminology proposed by Albuquerque et.al. (D. V. Albuquerque. J kottor and N. Velumurgan). Radiographically working length was determined. Radiograph showed that (5 canals) two in mesiobuccal and mesiolingual canals in mesial root and single canal in MB; one canal in distal root and one canal in distolingual root.

For shaping and cleaning, rotary ProTaper instruments were used in a step down manner. As a lubricant Glyde (Dentsply) was used and sodium hypochlorite, chlorhexidine and normal saline were used as irrigants. Canals were obturated using cold lateral condensation technique. Radiograph was taken after obturation. Postoperative (8 months) radiograph shows normal pdl space and there is no radiolucencies in the interradicular and periapical regions: then the tooth was restored with permanent restoration (crown) in 2 cases. In case 1 and case 2 contralateral radiograph reveals that 4 roots i.e. additional mesiobuccal (Radix paramolaris) and distolingual root (Radix entomolaris) on left side.

\section{Discussion}

The study of morphology of root and root canal has endodontic significance (Vertucci 1984). Any tooth may develop accessory roots. Variations in the morphology and anatomy of root and root canals in multi rooted teeth are a constant challenge for diagnosis and management. A precise radiographic system and legitimate translation are fundamental for sound analysis and treatment. The utilization of pre-operative radiographs at various angulations identifies and assess the root and root canal anatomy. 8 The present case report illustrates 4 rooted and 5 canals first mandibular molar with 2 mesial and 2 distal roots, the mesial root having mesiobuccal and mesiolingual canals and the other 3 (mesiobuccal, distal and distolingual) roots have an single separate root canals

\section{Prevalence of Radix Entomolaris and Radix Paramolaris}

The existence of RE in the first permanent mandibular molar is related with certain ethnic groups. Its predominance shifts in various population. For eg: In African population 3\% [8,9], In Eurasian and Indian populace less than 5\% [10], In Mongoloid traits which include Chinese Eskimos and American Indians it compasses from 5 - 30\%, In Caucasian population it ranges from $3.4-4.2 \%[11,12]$. Thus, RE is considered as Eumorphic root morphology in Mongoloid traits as its frequency of occurrence is high in these traits. Whereas in Caucasians it is considered as Dysmorphic root morphology as its frequency of occurence is low.

A Radix Entomolaris can be found on the permanent first, second and third mandibular molar eventuating least customarily on the second molar [13]. Although some studies have reported the occurrence of bilateral RE ranging from 50\%-67\% [14,15]. The frequency of Radix Entomolaris on the mandibular first molar for female patients $(6.92 \%)$ is relatively higher than that of male $(5.83 \%)$ patients. The distribution of RE on the right side is $2.4 \%$ whereas on the left it is $0.4 \%$. The left mandibular permanent first molar in male patients had minimal frequency of RE i.e., $0 \%$; the two-sided event of $\mathrm{RE}$ on first mandibular molar in female patients had the most elevated rate i.e., $3.85 \%$ [16].

Bolk16 reported the occurrence of additional buccal root: the RADIX PARAMOLARIS. The occurrence of RP is very rare when compared to RE. According to Visser13 the prevalence of Radix Paramolaris was found to be $0 \%$ for the first mandibular molar, $0.5 \%$ for the second mandibular molar and $2 \%$ for the third mandibular molar. However, other studies have reported RP in 1st mandibular molars $[7,9]$.

\section{Etiology}

The etiology behind the development of RE is as yet indistinct. In eumorphic roots, racial hereditary variables impacts the more significant expression of the specific gene that results in the more prominent phenotypic appearance [17-19]. In dysmorphic roots its origination could be associated with external factors amid odontogenesis or to polygenetic system.

\section{Morphology}

Normally, RE is smaller than the distal and mesial roots. The dimensions of RE can diversify from a short conical extension to a mature root with ordinary length and root canal. According to the location of the cervical part of RE, Carlsen and Alexandersen 6 describes four different types of RE. TypeA, B, C and AC. Types A and $B$ refer to a distally located cervical part of RE with two normal distal roots and one normal distal root respectively. Type $\mathrm{C}$ refers a mesially located cervical part and Type AC refers to a central location between 
mesial and distal root components. This classification allows for the diagnosis of separate and non-separate RE. A classification by De Moore and et al. [20] based on the curvature of the separate RE variants in the buccolingual orientation is of three types. Type 1 refers to a straight root/root canal. Type 2 refers to an initially curved entrance which continues as a straight root /root canal. Type 3 refers to an initial curve in the coronal third of the root canal and a second curve beginning in the middle third and continuing to the apical third.

Latterly, Song et al. grouped the RE into five types depending on their morphological characteristics [21] Type 1 refers to a straight root/ root canal. Type 2 refers to an initially curved entrance which continues as a straight root/root canal. Type 3 refers to an initial curve in the coronal third of the root canal and a second curve beginning in the middle and continuing to the apical third. Type 4 [small type], where the root length is less than half that of the distobuccal root and type 5 [conical type], which is a cone shaped extension with no root canal.

A classification by Carlsen and Alexandersen 6 described two different types of RP: Types A and B. Type A refers to a RP in which the cervical part is located on the mesial root complex; Type $B$ refers to a RP in which the cervical part is located centrally, between the mesial and distal root complexes. According to these classifications our cases belongs to type $-1 \mathrm{~A}$.

\section{Clinical Approach}

The presence of additional roots always have clinical insinuation in endodontic treatment. Hence an accurate diagnosis of these additional roots is essential to circumvent mishaps during endodontic treatment. An unambiguous diagnosis can be accomplished through a careful assessment of the preoperative radiographs followed by a proper elucidation. RE which is situated distolingually is usually indistinct because of the overlap of distal root over it, to find out the hidden RE an extra radiograph ought to be taken from a more mesial or distal angle (30 degrees).

A thorough clinical examination of the crown and cervical morphology of the roots by walking probe can facilitate the identification of an extra root. Existence of an extra cusp (Tuberculum paramolare), bulbous crown or more pronounced occlusodistal or distolingual lobe, in concoction with the cervical prominence or convexity indicates the nearness of an additional root. Hence, a thorough clinical inspection also plays a major role in identifying the presence of supernumerary root.

On the pulp chamber floor a dark line signifies the presence of an orifice. The position of orifices of the root canals of RE and RP has connotations for opening the cavity. The orifice of Radix Entomolaris located more distolingually to the main canal or canals of the distal root. An extension of the triangular opening cavity from mesiolingual to the distolingual root canal results in the trapezoidal outform which enhances the localization and access to the root canals. Visual aids (loupe, intraoral cameras, dental microscope and $\mathrm{CBCT}$ ) are also helpful in locating these canals.

\section{Conclusion}

Unvanquished endodontic treatment starts with appropriate clinical and radiographic inspection. The initial diagnosis of the supernumerary roots such as RE and RP before commencing the endodontic treatment is utmost necessary to avoid procedural mishaps. The mean interorifice space may help endodontists to unearth the orifices and also to modify the access preparations, which might enhance the success rate of an endodontic treatment. Therefore, a clinician must be keen-eyed as diversifications of root and root canal anatomy might be experienced at any time during the treatment procedures.

\section{References}

1. Slowey RR (1974) Radiographic aids in the detection of extra root canals. Oral Surg Oral Med Oral Pathol 37: 762-772.

2. Luciane F (1996) External and Internal Anatomy of Mandibular Molars. Braz Dent J 7: 33-40.

3. Burns RC, Herbranson EJ (2002) Tooth morphology and access cavity preparation in pathways of the pulp, (8th Edn), Mosby, St.Louis, USA, pp: 173-229.

4. Carabelli G (1844) Systematisches Handbuch der Zahnheilkunde, (2nd edn), Vienna: Austria, p: 114.

5. Bolk L (1915) Bemerkugen uber Wurzelvariationen am menschlichen unteren Molaren. Zeiting fur Morphologie und Anthropologie 17: 605-610.

6. Carlsen O, Alexandernsen V (1990) Radix Entomolaris; identification and morphology. Scan J Dent Res 98: 363-373.

7. Carlsen O, Alexandernsen v (1991) Radix Paramolaris in permanent mandibular molars:identification and morphology. Scan J Dent Res 99: 189-195.

8. Fava LRG, Dummer PMH (1997) Periapical radiographic teeth during diagnosis and treatment. Int Endod J 30: 250-261.

9. Sperber GH, Moreau JL (1998) Study of the number of roots and canals in Senegalese first permanent mandibular molars. Int Endod J 31: 112-116.

10. Tratman EK (1938) Three rooted lower molars in man and their racial distribution. Br Dent J 264-274.

11. Curzon ME (1973) Three rooted mandibular permanent molars in English Caucasians. J Dent Res 52: 181.

12. Feraz JA, Pecora JD (1993) Three rooted mandibular molars in patients of mangolian, Caucasian and negro origin. Braz Dent J 3: 113-117.

13. Visser JB (1948) Beitrag Zur Kenntinis der menschlichen Zahnwurzelformen. Hilversum: Rotting 49-72.

14. Steelman R (1986) Incidence of an accessory distal root on mandibular first permanent molars in Hispanic children. J Dent child 53:122-3.

15. Yew SC, Chan K (1993) A retrospective study of endodontically treated mandibular first molars in a Chinese population. J Endod 19: 471-473.

16. Garg AK, Tewari RK, Agarwal N (2013) Prevalence of three rooted mandibular first molar among Indians using SCT. Int Dent $\mathrm{J} 2$.

17. Bolk L (1914) Welcher Gebi Breihe gehoren die Molaren an. Z Morphol Antropol 1783-116.

18. Reichart PA, Metah D (1981) Three rooted permanent mandibular first molars in the Thai. Community Dent Oral Epidemiol 9: 191-192.

19. Ribeiro FC, Consolaro A (1997) Importanica clinicay antropologica de la raiz distolingual en las molars inferiors permanents. Endodoncia 15: 72-78.

20. De Moore RJ, Deroose CA, Calberson FL (2004) The radix entomolaris in mandibular first molars: an endodontic challenge. Int Endod J 37: 789-799.

21. Song JS, Choi HJ, Jung Y, Kim HSO (2010) The prevalence and morphogenic classification of distolingual roots in the mandibular molars in a Korean population. J Endodont 36: 653-657. 